\title{
Research Paper \\ Comparison of Changes in Center of Pressure and Time to Stabilization in Students With and Without Hyperlordosis
}

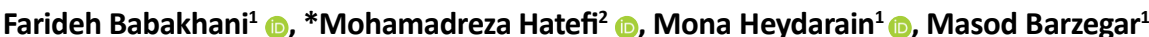

1. Department of Sports Pathology \& Corrective Exercises, Faculty of Physical Education and Sports Sciences, Allameh Tabataba'i University, Tehran, Iran.

2. Department of Biomechanics and Sport Injuries, Faculty of Physical Education and Sports Sciences, Kharazmi University, Tehran, Iran.

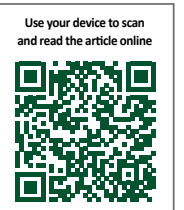

Citation: Babakhani F, Hatefi M, Heydarain M, Barzegar M. [Comparison of Changes in Center of Pressure and Time to Stabilization in Students with and without Hyperlordosis (Persian)]. Journal of Sport Biomechanics. 2018; 4(3):62-73. https://doi. org/10.32598/biomechanics.4.3.62

doi) https://doi.org/10.32598/biomechanics.4.3.62

Key words:

Force plate, Balance,

Postural control

\section{ABSTRACT}

Objective Considering the importance of balance in performing daily tasks and sports activities, it is necessary to examine the balance of the body from different angles. The aim of this study was to compare the changes in Center of Pressure (COP) and Time to Stabilization (TTS) in students with and without hyperlordosis.

Methods In this study, 30 female students of Tehran's middle school with a range of 14-15 years old who were selected by available sampling method were participated. Subjects' spinal curvature was evaluated by a flexible ruler. Which divided into two groups: 15 patients with hypereloidosis and 15 healthy subjects. To assess postural static stability in a static state, from open and closed eyes on the force plate (Ver 3.0.2 model from an Iranian scientist) was used. also to assess postural stability in the dynamic state, the landing stability test on the force plate device was used. Independent Sample T test statistics were used to analyze the data.

Results The results of the statistical test showed that there was no significant difference in the parameters of the center of pressure (COP) in two- position with open and closed eyes between the two groups of healthy and hyperlordosis $(P>0.05)$. But the time to stabilization (TTS) between the two healthy groups and hyperlordosis was statistically significant $(\mathrm{P}<0.05)$.

Conclusion The results of this study showed that the Spine Curvature Disorders (hyperlordosis) as a risk factor can distort the dynamic balance index (time to stabilization), But has no affect the static balance with both open eyes and closed eyes (center of pressure).

\section{Extended Abstract}

\section{Introduction}

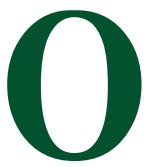

ne of the most common abnormalities in high school students is lumbar hyperlordosis [2]. Hyperlordosis is spinal deformity occurring on the sagittal plane; excessive lumbar curvature causes the forward dis- placement of the center of gravity and increases pelvic motion [3]. As a result of increased lumbar lordosis, the erector spinal muscles and hip flexors become shortened or stiff. Besides, the abdominal muscles and hip extensors become weak or stretched. Natural lordosis in the lumbar region protects it against excessive pressure and acts as a shock absorber $[4,5]$. Murray et al. compared balance between individuals with and without ankylosing spondylitis, a form of fixed kyphosis; they concluded that these patients have

\section{* Corresponding Author:}

Mohamadreza Hatefi

Address: Department of Biomechanics and Sport Injuries, Faculty of Physical Education and Sports Sciences, Kharazmi University, Tehran, Iran Tel: +98 (912) 2440394

E-Mail: hatefimohamadreza@yahoo.com 
more postural fluctuations, compared to healthy controls [15]. However, Aydog et al. reported no significant difference in postural fluctuations and the Center of Pressure (COP) between the two groups [16]. Spinal deformities may displace the center of gravity and affect balance. Thus, investigating the effect of spinal deformities can provide beneficial data on the balance function of these individuals. Such information could be applied to adopt effective methods to reduce the effects of these abnormalities on their ability to maintain balance, health promotion, and sports performance. Various methods are available to evaluate dynamic balance. Numerous researchers have used the Star Excursion Balance Test [18]; however, it is not as functional and dynamic as the jump-landing task required to measure Time to Stabilization (TTS) [19]. TTS assessment is the latest instrument for neuromuscular control measurement. TTS uses sensory and mechanical systems to perform complex jump-landing task. As a result, it indicates the body's ability to minimize postural fluctuations when transferring from a dynamic position to a static state [20]. It is also very sensitive to postural stability disorders [21]. This study aimed to compare the changes in COP and TTS between students with and without hyperlordosis.

\section{Participants and Methods}

In total, 30 female high school students in Tehran Province, Iran, aged 14-15 years, participated in this study. They were selected by a convenience sampling technique and based on the study inclusion criteria. They were divided into two groups of patients $(n=15)$ and controls $(n=15)$. The subjects' lumbar spine curvature was evaluated by a $30-\mathrm{cm}$ Iranian-made flexible ruler like the French curve; its' reliability and validity have been reported as $98 \%$ and $82 \%$, respectively, in Iran. COP parameters to examine the balance in double-leg standing position with eyes open and closed included the following: the range of variation (R), Path Length (PL), Standard Deviation (SD), and Root Mean Square (RMS) in Anterior-Posterior (AP) and Mediolateral (ML) directions; average radial displacement (RDavg), standard deviation of RDavg, Total Path Length (TPL), as well as the ellipse surface area of base of support (CEA). The assessments of changes in COP were performed in 30 seconds with eyes open and closed. Foot COP information was recorded in lateral and anterior-posterior directions by force plate $[22,24]$.

To measure TTS in three directions of vertical, AP, and $\mathrm{ML}$, the study participant was requested to stand on a

Table 1. T-test results for comparing COP variables in a double-leg standing position with eyes open

\begin{tabular}{|ccccc}
\hline COP Variables & Control Group & Patient Group & t & Sig. \\
\hline R. AP $(\mathrm{mm})$ & $04 . \pm 94.6$ & $62.4 \pm 20.5$ & 099.1 & 281.0 \\
\hline R. ML $(\mathrm{mm})$ & $62.1 \pm 59.2$ & $95.0 \pm 38.1$ & 510.2 & 81.0 \\
\hline PL. AP $(\mathrm{mm})$ & $05 . \pm 09.42$ & $2.19 \pm 61.36$ & 784.0 & 440.0 \\
\hline PL. ML $(\mathrm{mm})$ & $22.10 \pm 96.38$ & $97.11 \pm 09.39$ & 034.0 & 974.0 \\
\hline V. AP $(\mathrm{mm} / \mathrm{s})$ & $95 . \pm 10.2$ & $96.0 \pm 83.1$ & 784.0 & 440.0 \\
\hline V. ML $(\mathrm{mm} / \mathrm{s})$ & $51.0 \pm 95.1$ & $59.0 \pm 95.1$ & 034.0 & 974.0 \\
\hline RDavg $(\mathrm{mm})$ & $59.1 \pm 20.6$ & $65.1 \pm 18.5$ & 726.1 & 095.0 \\
\hline CEA $(\mathrm{mm})$ & $15.96 \pm 03.196$ & $55.68 \pm 92.144$ & 676.1 & 105.0 \\
\hline SD. AP $(\mathrm{mm})$ & $86.0 \pm 86.1$ & $6.0 \pm 61.1$ & 913.0 & 369.0 \\
\hline SD. ML $(\mathrm{mm})$ & $74.1 \pm 87.5$ & $76.1 \pm 90.4$ & 527.0 & 138.0 \\
\hline SD. RDavg $(\mathrm{mm})$ & $36 . \pm 60.0$ & $54.0 \pm 46.0$ & 841.0 & 408.0 \\
\hline TPL $(\mathrm{mm})$ & $32.22 \pm 44.64$ & $98.23 \pm 67.59$ & 564.0 & 577.0 \\
\hline RMS. AP $(\mathrm{mm})$ & $32.0 \pm 71.0$ & $38.0 \pm 53.0$ & 460.1 & 155.0 \\
\hline RMS. ML $(\mathrm{mm})$ & $14 . \pm 47.0$ & $26.0 \pm 41.0$ & 701.0 & 489.0 \\
\hline V $=$ velocity & & & & Journal of \\
\hline
\end{tabular}


40-cm-height stair distanced $70 \mathrm{~cm}$ from the center of the force plate [25]; then, land on the center of the force plate with one foot (the dominant foot). Accordingly, as soon as positioned, the si=subject must have placed the hands on the pelvic area, hold the head up, and look forward while trying to maintain balance. Before that, the study subjects were requested to hit the ball to determine their dominant foot $[26,27]$.

\section{Results}

There was no significant difference between the two groups in any variables of COP when standing with eyes open (Table 1) and closed. In some cases, the variables were slightly different; however, this difference was not statistically significant $(\mathrm{P}>0.05)$. Regarding TTL, there was a significant difference between the study groups in three directions of vertical, AP, and ML. In those with hyperlordosis, the TTL was longer than that of the controls $(\mathrm{P}<0.05)$.

\section{Discussion}

The present study results suggested no significant difference in foot COP variables in standing with eyes closed and open between two groups of healthy and hyperlordosis individuals. However, the TTS result was significantly different between the two study groups. Physical posture is always in a state of fluctuation, and its balance is maintained by the combined reactions of visual, proprioceptive, and vestibular senses data [28]. Based on the literature, COP displacement has been used as an indirect attribute of postural fluctuation and one's ability to maintain balance and postural control, consequently [29].

\section{Conclusion}

Abnormal spine alignment (hyperlordosis), as a risk factor, can affect dynamic balance index (i.e. TTS); however, it has no effect on the static balance index (i.e. COP) with eyes open and closed. Therefore, the improvement of hyperlordosis deformity may improve postural control in dynamic balance indices.

\section{Ethical Considerations}

\section{Compliance with ethical guidelines}

All ethical principles were considered in this article. The participants were informed about the purpose of the research and its implementation stages; they were also assured about the confidentiality of their information; Moreover, They were allowed to leave the study whenever they wish, and if desired, the results of the research would be available to them.

\section{Funding}

This research did not receive any specific grant from funding agencies in the public, commercial, or not-forprofit sectors.

\section{Authors' contributions}

All authors contributed equally in preparing all parts of the research.

\section{Conflicts of interest}

The authors declared no conflict of interest. 


\title{
مقايسه تغييرات مركز فشار و زمان رسيدن به يايدارى در دانشآموزان با وبدون هايبرلوردوزيس
}

\author{
فريده باباخانى' هـ "محمدرضا هاتفى' ه، مونا حيدريان' هـ مسعود برز5ر' \\ 1. كروه آسيبشئاسى و حركات اصلاحى، دانشكده تربيتبلنى و علوم ورزشي، دانشعاه علامه طباطبائى، تهران، ايران.

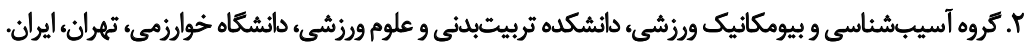

\begin{abstract}
حكين

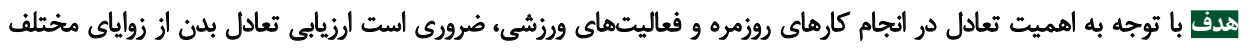

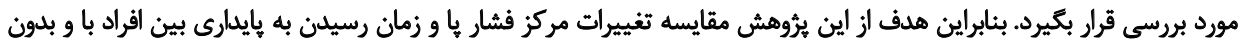
هايثرلوردوزيس بود.

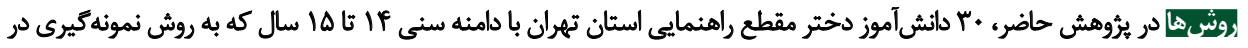

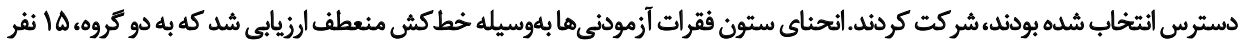

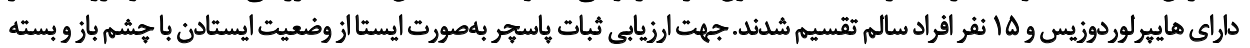

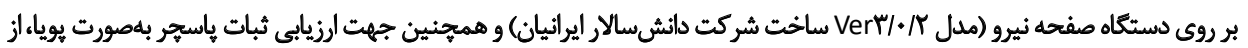

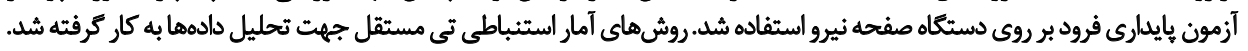

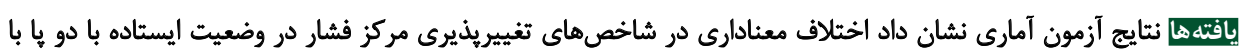

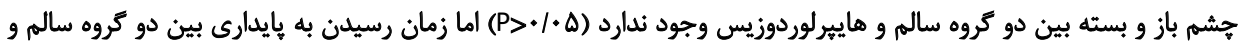

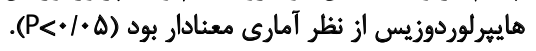

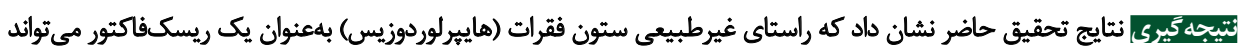

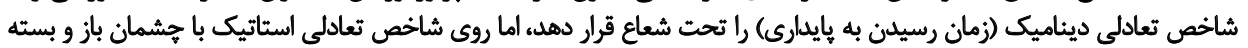

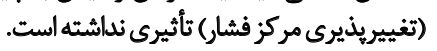

اطلاعات مقاله:

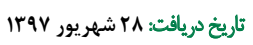

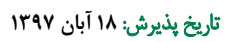

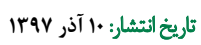

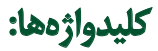

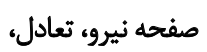

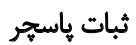

فشارهاى بيش از حد در اين ناحيه محافظت مى كند و بهن بهنوان

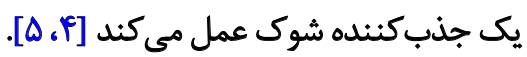

مقدمه

همجنين ناهنجارىهاى ستون فقرات كه به دلايل ارثي،

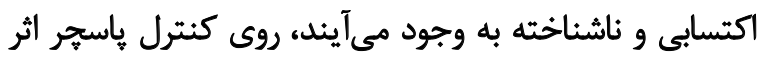

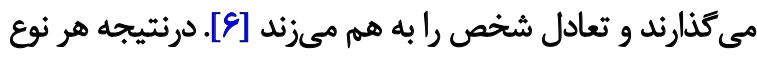

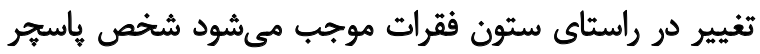

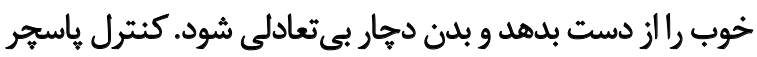

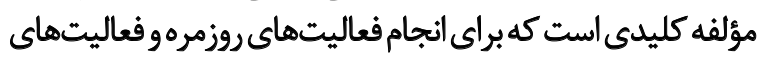

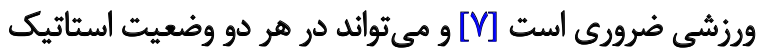

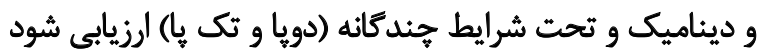

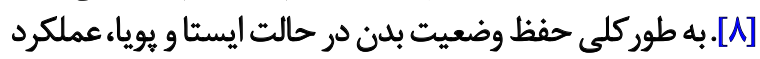

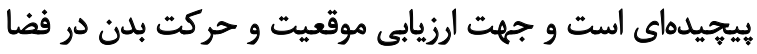

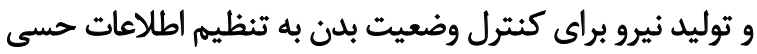

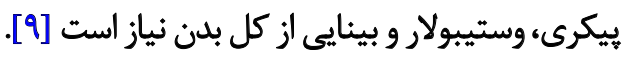

سلامت جسمانى و داشتن وضعيت بدنى مطلوب در زندكى

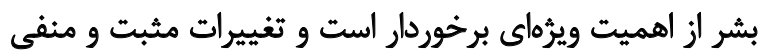

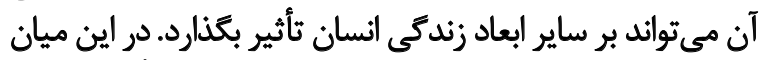

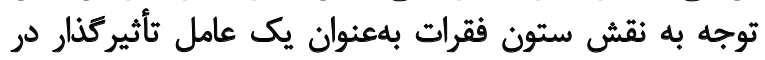

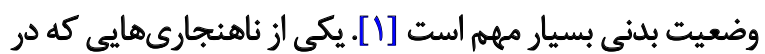

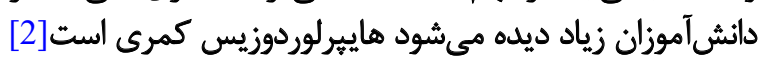

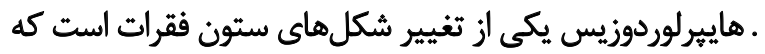

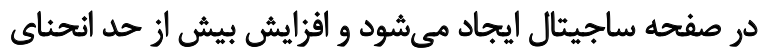

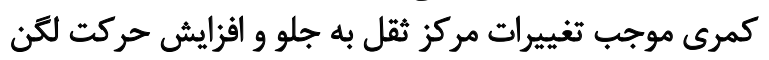

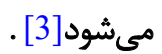
در افزايش تودى كمر عضلات اركتور اسياين و فلكسورهاى

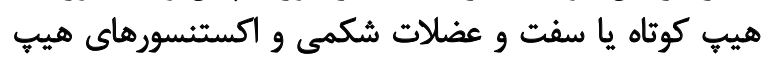

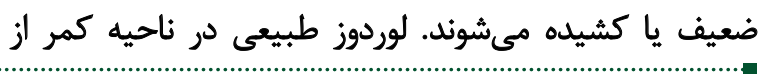

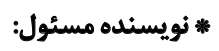

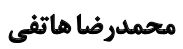
نشاني: كروه آسيبشناسي و حركات اصلاحي، دانشكده تربيتبدنى و علوم ورزشي، داتشكاه خوارزمي، تهرانء ايران.

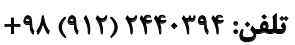
يست الكترونيكي: بلفي 
افراد مبتلا به دفورميتى ستون فقرات، علاوه بر اندكبودن، كاهيى

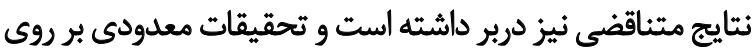
كنترل ياسجحر در افراد لوردوزيس متمركز شدهاند.

با توجه به اينكه ناهنجارىهاى ستون فقرات ممكن است

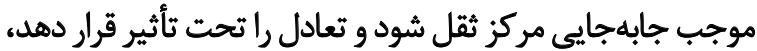

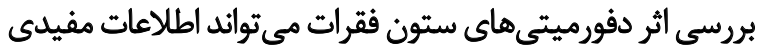

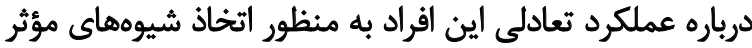

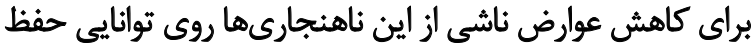

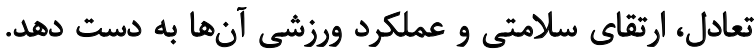

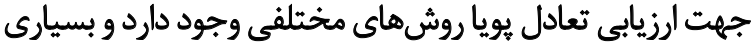

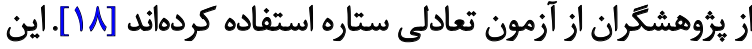

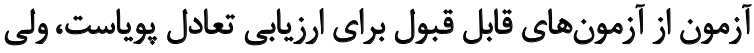

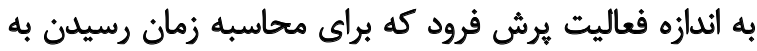

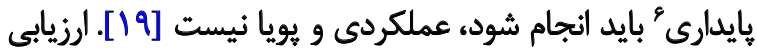

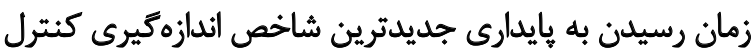

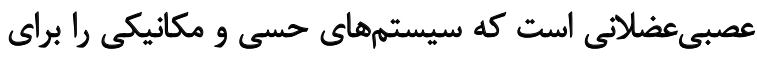

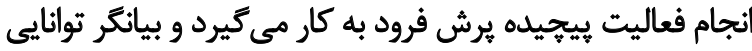

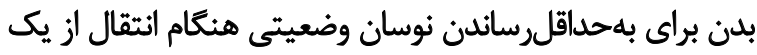

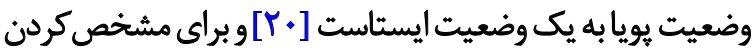

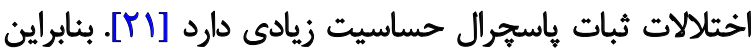

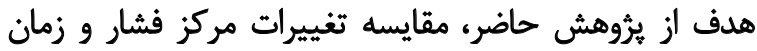
رسيدن به بايدارى بين افراد باو بدون هايير لوردوزيس است.

$$
\text { روش شُشاسي }
$$

در ثروهش حاضر، · ب دانشآموز دختر مقطع راهنمايى استان

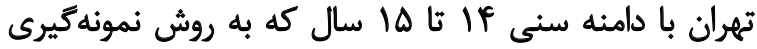

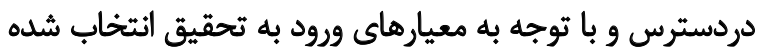

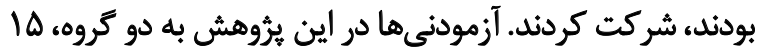

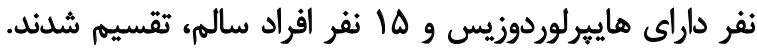

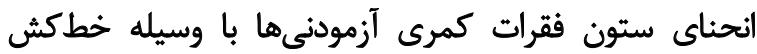

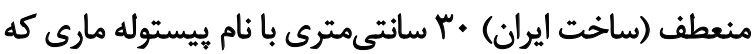

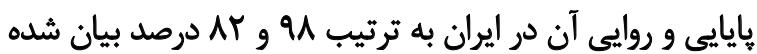

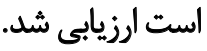

روش اندازهيرى زاويه لوردوز كمرى توسط خط ائى من منعطف

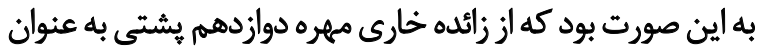

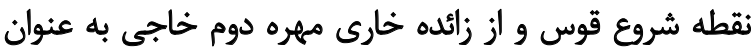

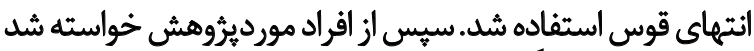

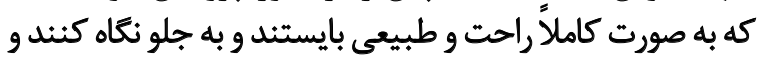

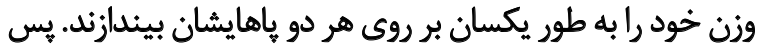

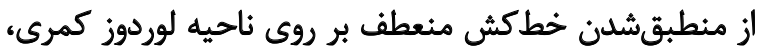

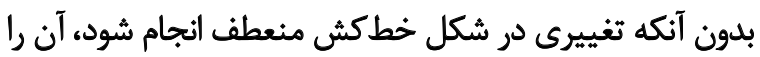

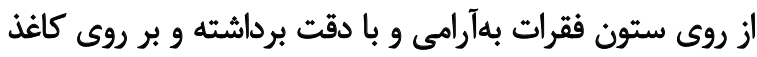

مهمترين كروههايى كه ضعف تعادل موجب آسيبديدكى آنها

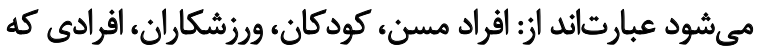

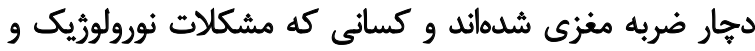

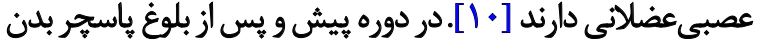

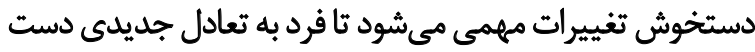

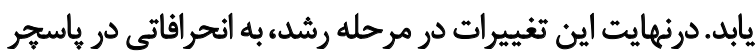

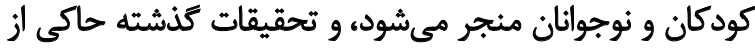
شيوع بيشتر ناهنجارى هايير لوردوزيس در سني

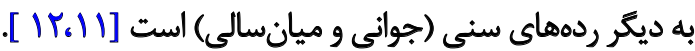

تاكنون مطالعات مختلفى در حيطههاى علوم رفتارى و

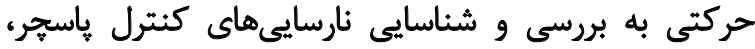

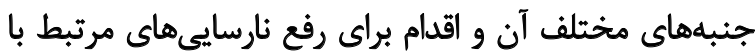

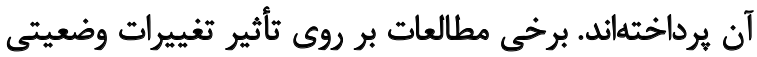

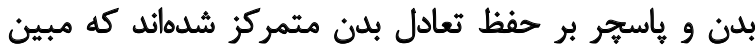

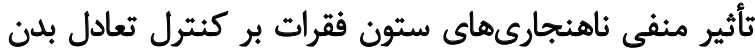

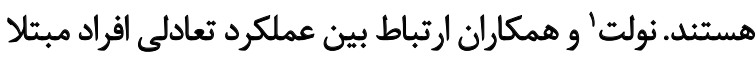

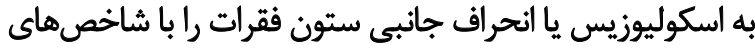

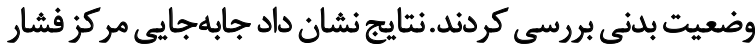

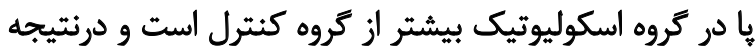

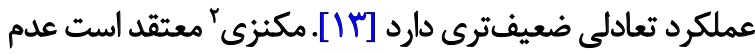

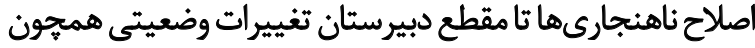

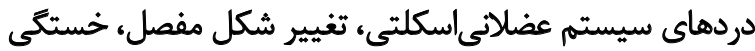

عضلانى و برهمخوردن تعادل را به همراه خواهد داشت [1 [1 ].

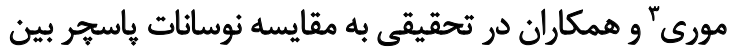

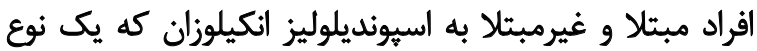

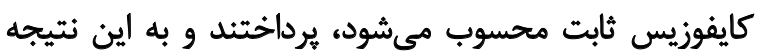

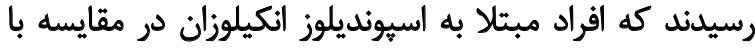

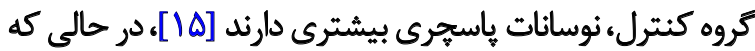

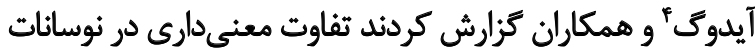

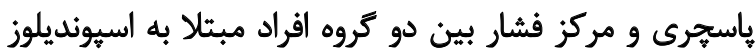

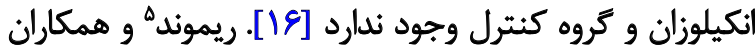

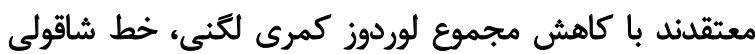

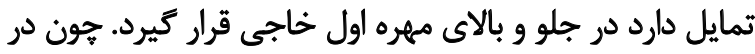

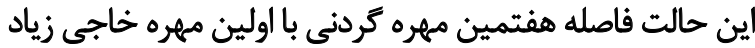

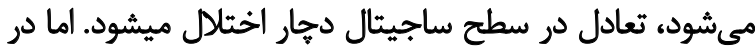

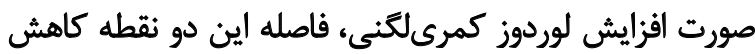

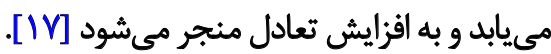
به طور كلى، مطالعات انجام كرفته در مورد كنترل ياسجر در

1. Nault

2. Mackenzie

3. Murray

4. Aydog

5.Raymond 


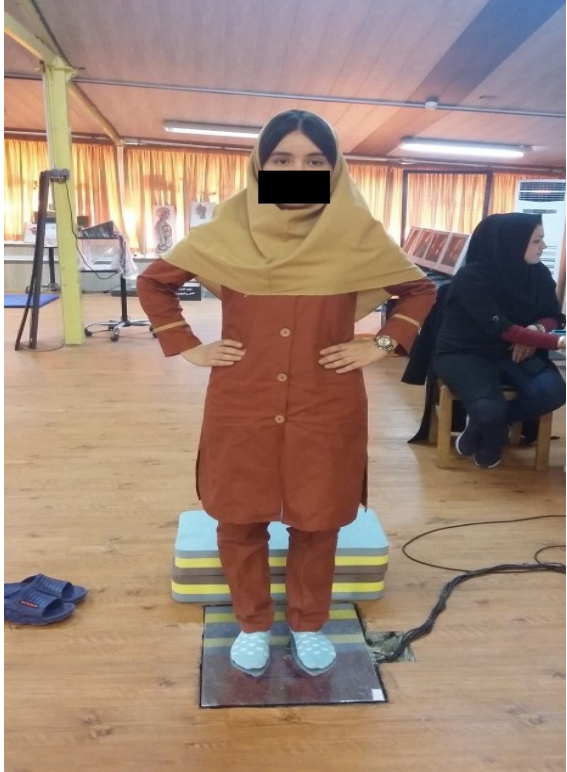

مجله بيومكانيك ورنث

\section{نحوه ارزيابى تغييرات مركز فشار}

شاخصهاى تغييريذيرى مركز فشار جهت بررسى تعادل

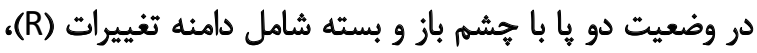

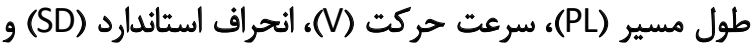

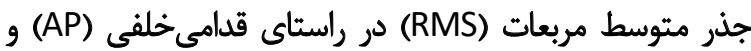

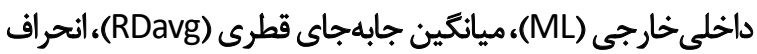

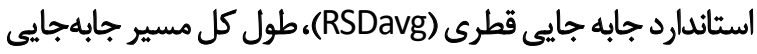

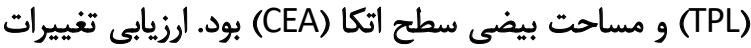

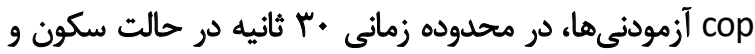

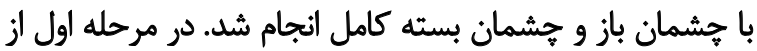

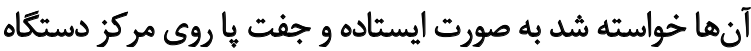

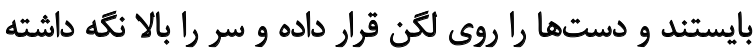

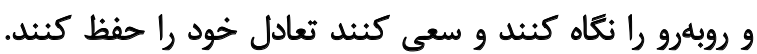

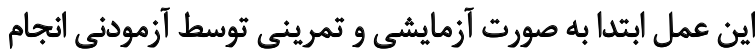

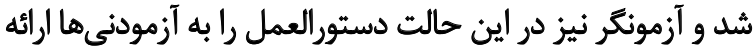

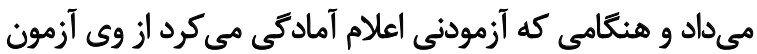

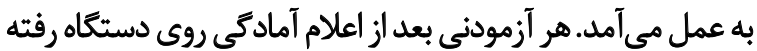

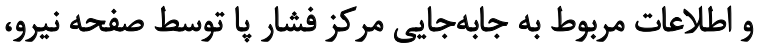

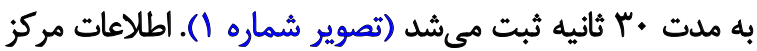

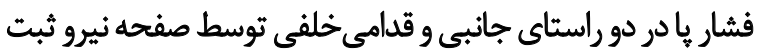

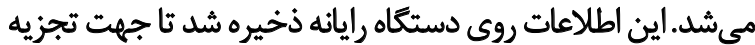

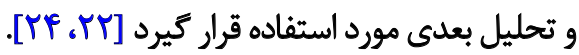

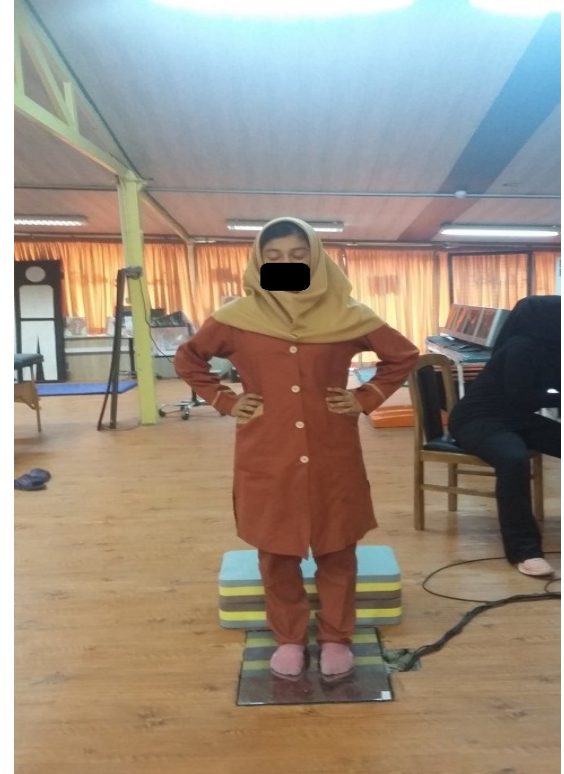

$$
\begin{aligned}
& \text { تصوير ا. ارزيابى تغييرات } \\
& \text { الف: مركز فشار با جششمان باز و بسته؛ } \\
& \text { بإ: آزمودنى هـا }
\end{aligned}
$$

سفيد، انحناى آن ترسيم شد. براى محاسبه زاويه قوس كمرى

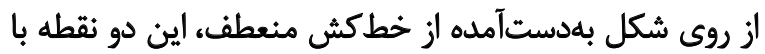

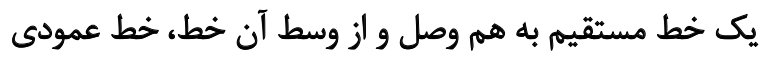

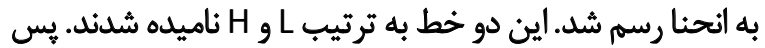

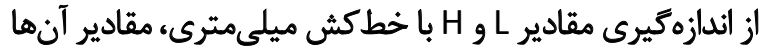

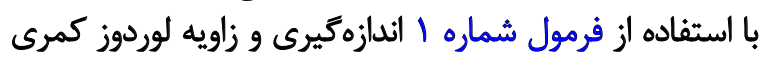

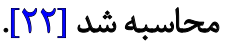

\section{1. $\vartheta=4 \operatorname{Arctan}[2 H / L]$}

همجينين آزمودنىها به صورت داوطلبانه فرم رضايتنامه

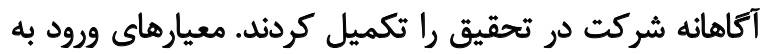

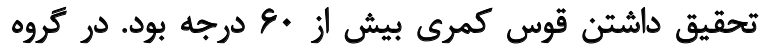

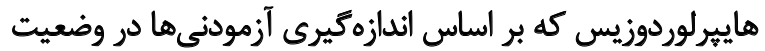

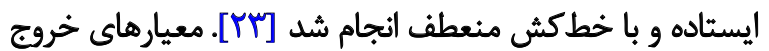

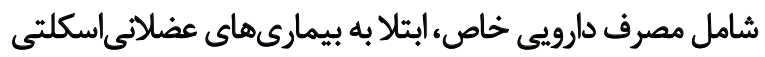

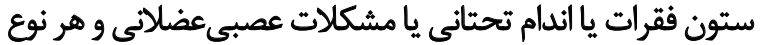

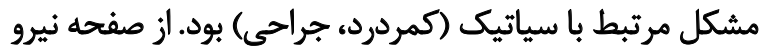

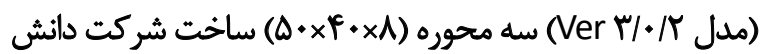

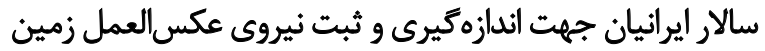

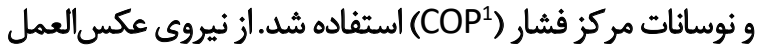

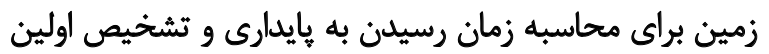

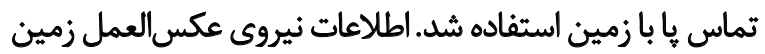

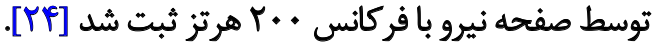




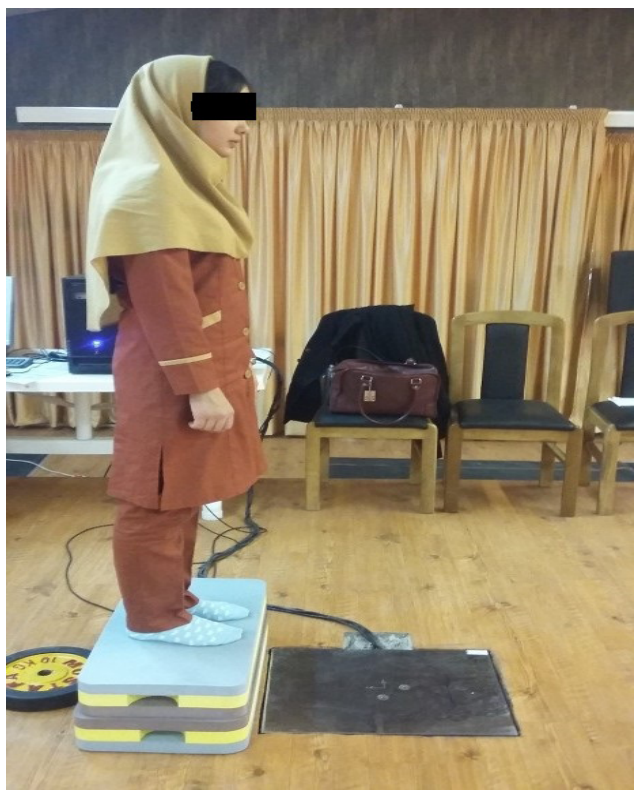

مجله بيومكانيك وزنش

توزيع دادها از آزمون كولموكروفاسميرنوف استفاده شد، لذا، به

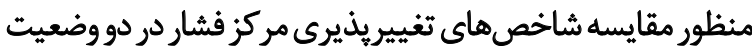

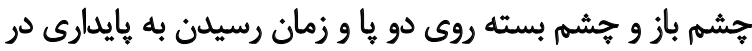

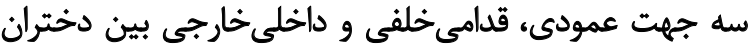

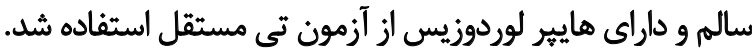

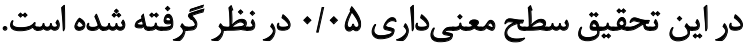

ثتايج

از آمار توصيفى (ميانگينثانحراف استاندارد) جهت بررسى إنى

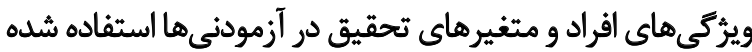

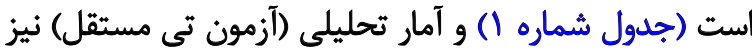
مورد استفاده قرار كرفته است

در هيجيجدام از شاخصهاى تغييريذيرى مركز فشار با دا در

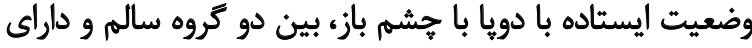

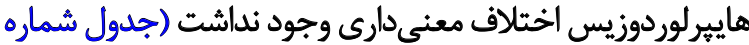

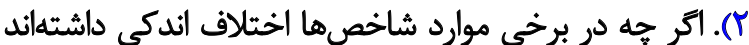

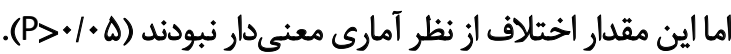

همجنين نتايج نشان داد شاخصهاى تغييريذيرى مركز فشار در

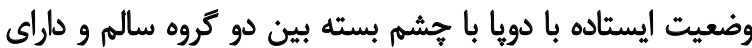

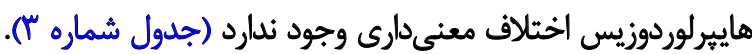

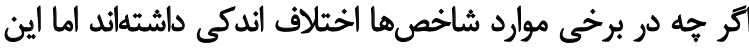

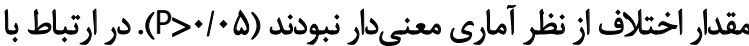

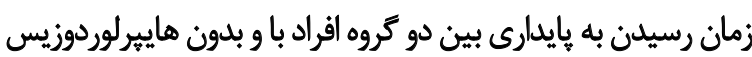

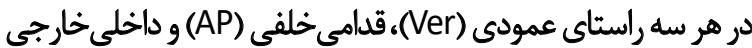
(ML)

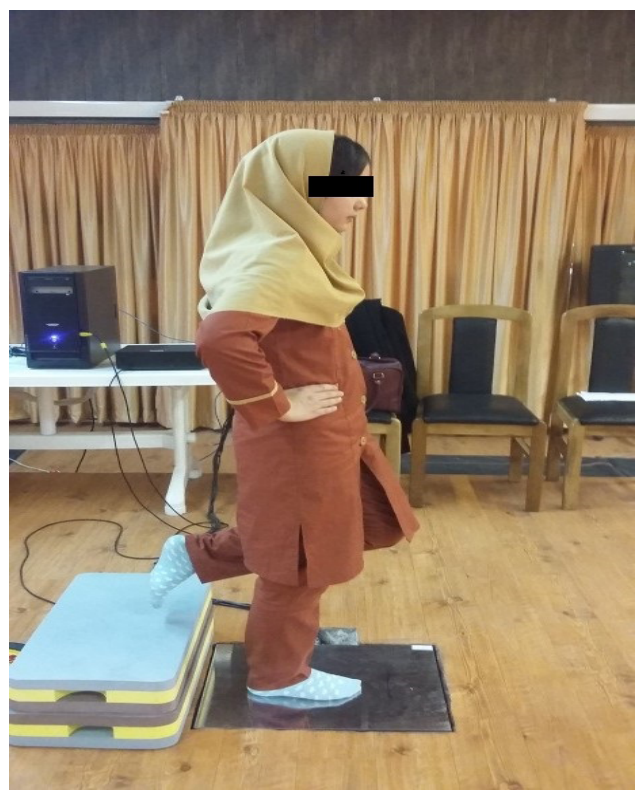

الف

تصوير لا. ارزيابى زمان رسيدن به يايدارى آزمودنىها

\section{نحوه ارزيابى زمان رسيدن به يإيدارى}

جهت بررسى زمان رسيدن به يايدارى در سه راستاى عمودى (MP)

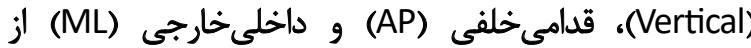

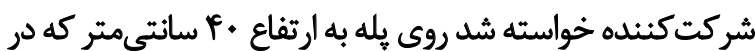

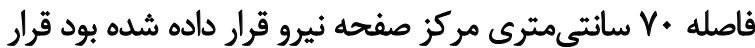

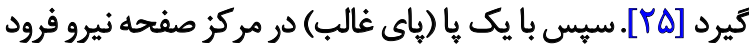

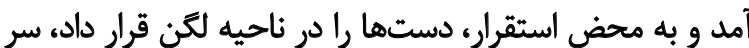

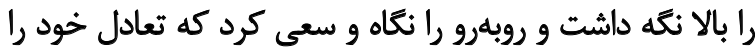

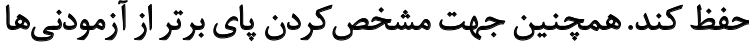

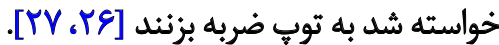

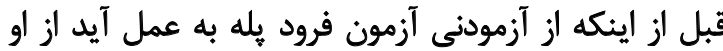

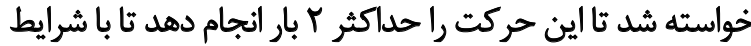

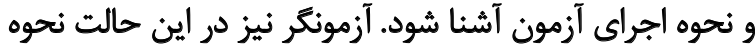

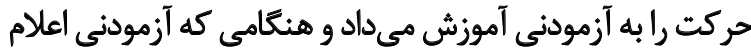

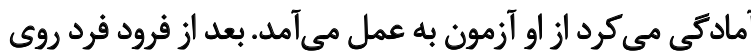

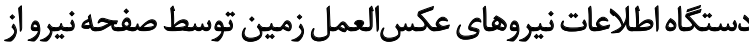

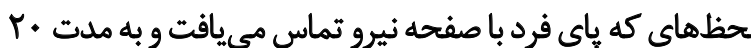

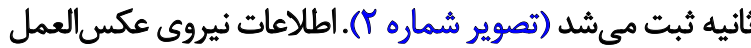

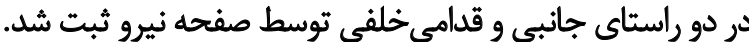

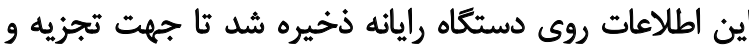
تحليل بعدى مورد استفاده قرار كيرد.

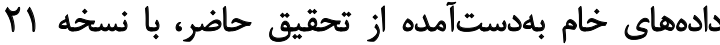

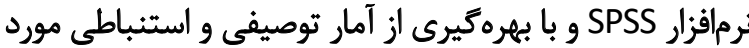

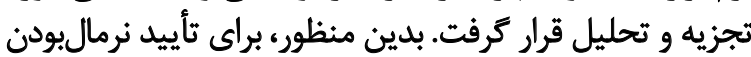


جدول ا. توصيف خصوصيات آنترويومتريكى نموئهاى موردمطالعه

\begin{tabular}{|c|c|c|c|c|}
\hline \multicolumn{3}{|c|}{ ميانكين+انحراف استاندارد } & \multirow{2}{*}{ تعداد } & \multirow{2}{*}{ توروه } \\
\hline وزن (كيلوكرم) & قد (سانتىمتر) & سن (سال) & & \\
\hline$\Delta V / \Delta F \pm \Delta / \Delta r$ & $\mid \Delta T / / V \pm F / T r$ & $|F /|+ \pm F \mid \cdot \Delta$ & is & 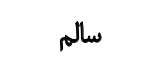 \\
\hline$\Delta H / W \pm \& / Y A$ & $\mid \Delta T / \cdot A \pm \Delta / P F$ & $|f / F V \pm F / T|$ & 10 & هاييرلوردوزيس \\
\hline
\end{tabular}

مجله بيومكانيك ورنش

با هشم باز و بسته بين دو گروه سالم و هاييرلوردوزيس

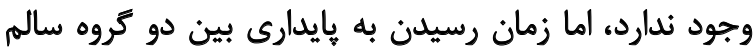

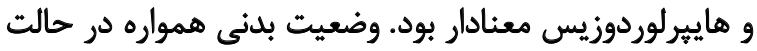

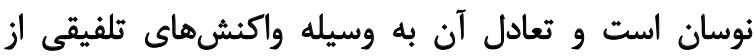

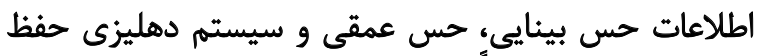

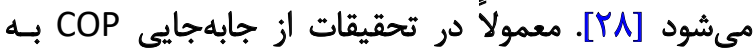

در دختران داراى هاييرلوردوزيس به طور معنى دارى بيشتر از زمان

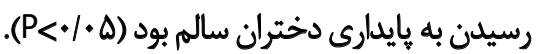

$\stackrel{\infty}{*}$

نتايج حاصل از تحقيق حاضر نشان داد اختلاف معنادارى در تغييريذيرى مركز فشار خا در وضعيتهائ ايستاده با دويا اختان جدول r. نتايج آزمونهاى تى مستقل جهت بررسى شاخصهاى تغييريذيرى مركز فشار در وضعيت ايستاده با دو با با جشم باز بين دو كروه سالم و داراى هاييرلوردوزيس

\begin{tabular}{|c|c|c|c|c|}
\hline \multicolumn{4}{|c|}{ ميانكين+|نحراف استاندارد } & \multirow{2}{*}{ شاخصهاى تغييريذيرى مركز } \\
\hline $\mathbf{T}$ & Sig. & كروه هايير لوردوزيس & كمروه سالم & \\
\hline $1 / .99$ & $\cdot / 4 \Lambda \mid$ & $\Delta / r \cdot \pm r / q r$ & $q / q \varphi \pm r / \cdot r$ & R. AP (mm) \\
\hline$\cdot / 4$ & $\Lambda 1 / \Delta 1$. & $1 / \Upsilon \Lambda \pm \cdot / 9 \Delta$ & $r / \Delta Q \pm 1 / \varepsilon r$ & R.ML (mm) \\
\hline$\cdot / v \wedge r$ &.$/ \mu F$ & $r q / 91 \pm 19 / r$ & $F T / \cdot Q \pm 19 / \cdot \Delta$ & PL.AP (mm) \\
\hline.$/ \cdot m e$ &.$/ 9 V^{4}$ & 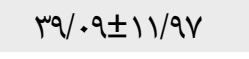 & $r N Q q \pm 1 \cdot / r T$ & PL.ML (mm) \\
\hline . VArF &.$/ \mu F$ & $1 / \Lambda \Psi \pm \cdot / q \varepsilon$ & $T / 1 \cdot \pm \cdot / 90$ & $\operatorname{V.AP}(\mathrm{mm} / \mathrm{s})$ \\
\hline . &.$/ 9 V^{c}$ & 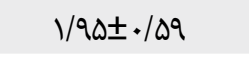 & $1 / 9 \Delta \pm \cdot / \Delta 1$ & V.ML $(\mathrm{mm} / \mathrm{s})$ \\
\hline I/VTE & .1 .90 & $\Delta / \backslash \pm \pm \mid / \varepsilon \Delta$ & $g / T \cdot \pm 1 / \Delta q$ & RDavg (mm) \\
\hline 1/9VG & $\cdot 11 \cdot 0$ & $\mid f \varphi / q \tau \pm \xi N \Delta \Delta$ & $\mid q \varepsilon / \cdot r \pm q \varepsilon / 10$ & CEA (mm2) \\
\hline (191/. & 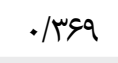 & $\mid / 91 \pm . / 8$ & $1 / N \varepsilon \pm \cdot / N \varepsilon$ & $\mathrm{SD} . \mathrm{AP}(\mathrm{mm})$ \\
\hline . /OTV & 吾 & $\varepsilon / q . \pm 1 / v \varepsilon$ & $\Delta / A \vee \pm I / V^{c}$ & SD.ML (mm) \\
\hline - $|\lambda F|$ & $\cdot / 4 \cdot 1$ & $\cdot / \& \& \pm \cdot / \Delta F$ & ع & SD.RD (mm) \\
\hline 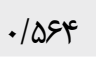 &.$/ \Delta W$ & $\Delta Q / g V \pm r r / Q \Lambda$ & $G T / A Y \pm T r / r T$ & $\mathrm{TPL}(\mathrm{mm})$ \\
\hline $1 / 4 \varepsilon$ &.$/ 1 \Delta \Delta$ & 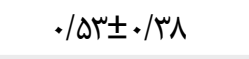 & rr/. & RMS.AP (mm) \\
\hline$\cdot / v \cdot 1$ &.$/ 4 \wedge 9$ & ./LID./4\& & $. / F V \pm \cdot / / F$ & RMS.ML (mm) \\
\hline
\end{tabular}

مجله بيومكانيك وزنش

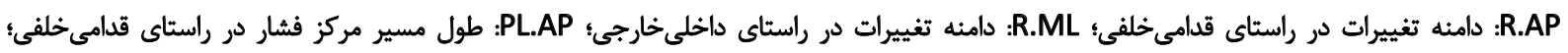

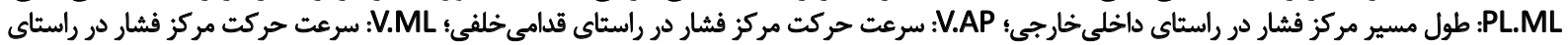

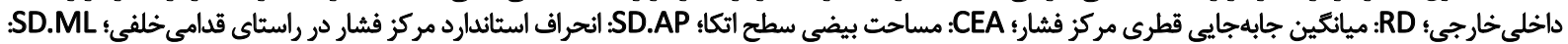

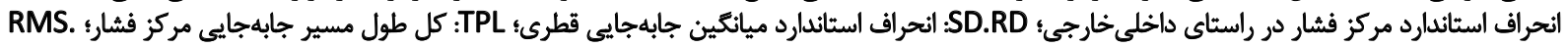

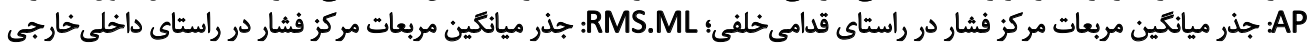


جدول ץ. نتايج آزمونهاى تى مستقل جهت بررسى شاخصهاى تغييريذيرى مركز فشار در وضعيت ايستاده با دو يا با جشم بسته بين دو كروه سالم و داراى هاييرلوردوزيس

\begin{tabular}{|c|c|c|c|c|}
\hline \multicolumn{4}{|c|}{ ميانكين||نحراف استاندارد } & \multirow{2}{*}{ شاخصهاى تغييريذيرى مركز } \\
\hline $\mathbf{T}$ & Sig. & كروه هايبرلوردوزيس & 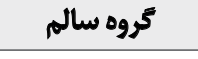 & \\
\hline $1 / \pi \wedge \Delta$ &,$\cdot / 1 W$ & $\mid \varepsilon / \varepsilon \notin \pm \Delta / \cdot q$ & $\mid f / \Delta \Psi \pm \Psi / \cdot \Lambda$ & R. AP (mm) \\
\hline$\cdot / \pi \Delta \Delta$ & , & $T / V \cdot \pm T / W$ & $r / 4 T \pm . / Q \Lambda$ & R.ML (mm) \\
\hline 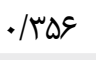 &,$\cdot / V T \Delta$ & $V V / \Lambda q \pm r / \Delta T$ & $s q / r+ \pm r \cdot 1 \cdot 1$ & PL.AP (mm) \\
\hline •/TAY &,$\cdot / \mathrm{mr}$ & $\Delta N\| \pm\| / \times q$ & $\Delta F / \wedge ৭ \pm I) / \Delta \Lambda$ & PL.ML (mm) \\
\hline • $/ \mu \Delta S$ &,$\cdot / N \Delta$ & $r / \Delta q \pm V / \cdot V$ & r/אצII & $\operatorname{V.AP}(\mathrm{mm} / \mathrm{s})$ \\
\hline$\cdot /$ TAV &,$\cdot / m$ & $r / 9) \pm \cdot / \Delta V$ & $r / \Lambda \uparrow \pm \cdot / \Delta V$ & V.ML $(\mathrm{mm} / \mathrm{s})$ \\
\hline ./VTA & ./FVA & $\varphi / \Delta \Delta \pm r / \cdot \varphi$ & $4 / \cdot 1 \pm r$ & $\operatorname{RDavg}(\mathrm{mm})$ \\
\hline •/А९ा & $\cdot /$ rVq & $r \wedge q / V \pm I r / / V$ & $r \Delta F / I V \pm 9 \Delta / r$ & $\mathrm{CEA}(\mathrm{mm} 2)$ \\
\hline 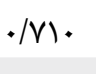 & $\cdot \mid r A F$ & $\Gamma / \mathcal{F} \Delta \pm \cdot / \mathcal{A}$ & $r / \Gamma \cdot \pm \cdot(\Delta)$ & $\mathrm{SD} . \mathrm{AP}(\mathrm{mm})$ \\
\hline . & . LTH & $9 / \pi) \pm r / \cdot 9$ & $\Delta / \wedge \Delta \pm 1 / \vee G$ & SD.ML (mm) \\
\hline.$/ \Delta Q F$ & $\cdot / \Delta \Lambda F$ & $r / r q \pm r / \Delta \Delta$ & $T / Y \Lambda \pm T / \mathcal{A} T$ & $\mathrm{SD} . \mathrm{RD}(\mathrm{mm})$ \\
\hline ERG &.$/ 8 V \pi$ & $1 . r / \mathcal{E} I \pm T \Delta / \varepsilon T$ & $q Q / \Delta \cdot \pm \Gamma r / \Delta q$ & $\mathrm{TPL}(\mathrm{mm})$ \\
\hline$. / 1+9$ & . MMr & $1 / \kappa+ \pm . / \varepsilon q$ & $1 / \kappa^{\mathcal{L}} \mid \pm \cdot / \tilde{\omega}$ & RMS.AP (mm) \\
\hline . KEA & •/Vq & $. / 91 \pm \cdot / 0$ & $. / 99 \pm . / 99$ & RMS.ML (mm) \\
\hline
\end{tabular}

مجله بيومكانيك وزنش

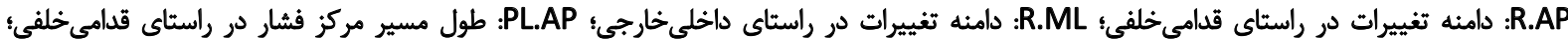

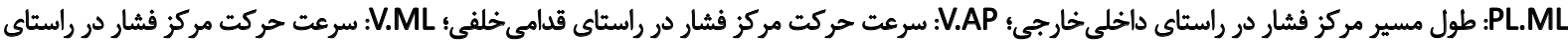

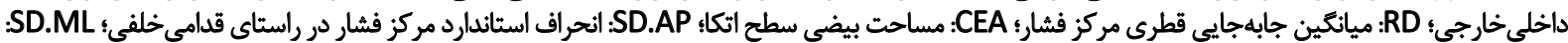

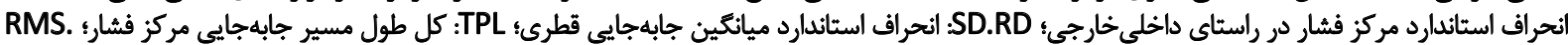

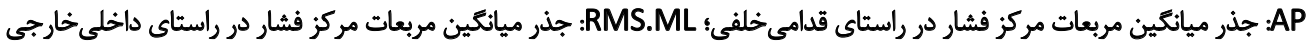

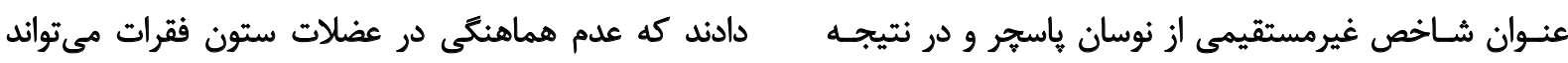

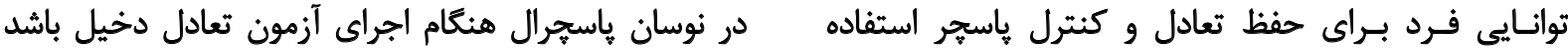

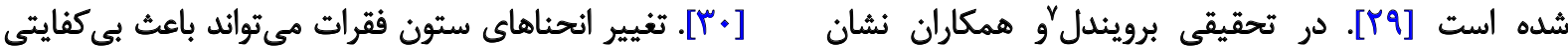

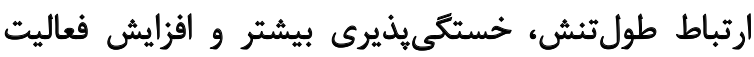

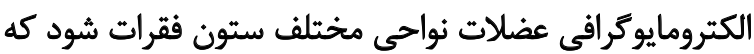

7. Bruyneel

جدول F. نتايج آزمونهاى تى مستقل براى مثايسه زمان رسيدن به بايدارى بين دو كروه باو بدون هاييرلوردوزيس

\begin{tabular}{|c|c|c|c|c|}
\hline \multirow{2}{*}{$\mathbf{T}$} & \multirow{2}{*}{ Sig. } & \multicolumn{2}{|c|}{ ميانكين ثلانحراف استاندارد } & \multirow{2}{*}{ راستاى زمان رسيدن به بايدارى } \\
\hline & & يروه هايير لوردوزيس & تروه سالم & \\
\hline $.1 . .1$ & , V/APA & $1 /$ rav $\pm \cdot / 10 V$ & $. / 9 Y Y \pm . / M V^{2}$ & (s) عمودي (s) \\
\hline $.1 . .1$ & $\Delta /\{1 \mid$ & $1 / n V \pm \cdot / r \cdot V$ & I/TAEt./Mer & قدامى خلفى (s) \\
\hline $.1 .+1$ & f/Rre & $I / R Y I \pm \cdot / T V E$ & $1 / .89 \pm+/ 1 n$ & داخلى خارجى (s) \\
\hline
\end{tabular}


بررسى اثر هايير كايفوزيس بر تعادل ايستا و يويا در دانشآموزان

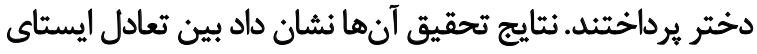

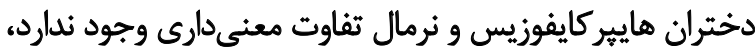

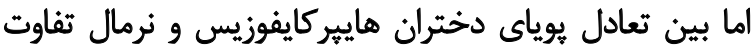

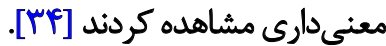

\section{نتيجهيَيرى نهايى}

يافتههاى برؤهش حاضر نشان داد راستاى غيرطبيعى ستون

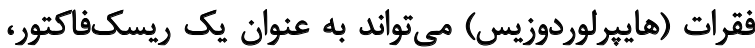

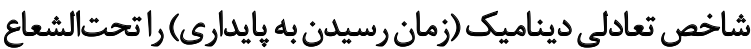

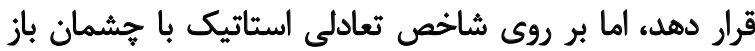
و بسته (تغييريذيرى مركز فشار) تأثيرى نداشته استي است. بنابراين بانين

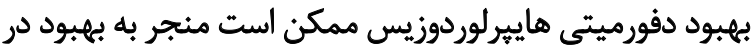
كنترل ياسجر در شاخص هائ تعادلى ديناميك شودد.

ملاحظات اخلاقى ييروى از اصول اخلاق يثروهش

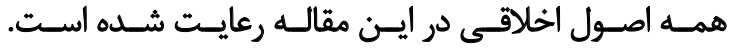

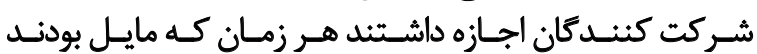

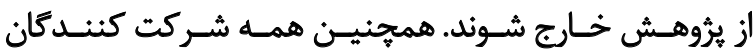

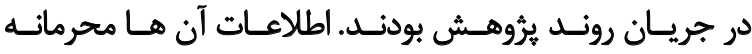

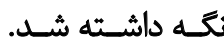

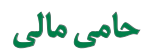

اين ثيروهش هيج كونه كمك مالى از سازمانىهاى دولتى، خصوصى و غيرانتفاعى دريافت نكرده است.

$$
\text { مشاركت نويسند }
$$

تمـام نويســندكان در آمادهسـازى ايــن مقالـه مشـاركت داشـــتهاند.

تعارض مناقع

بنابر اظهار نويسندكان، اين مقاله تعارض منافع ندارد.
اين افزايش فعاليت عضلانى به نوبه خود مي تواند منجر به

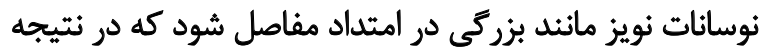

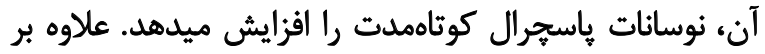

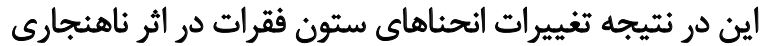

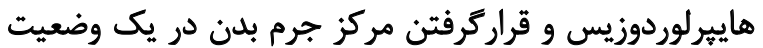

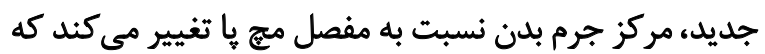
باعث تغيير در تشتاور مج ها و و افزايش فعاليت عضلات اندات اندام

تحتانى مى شود [آس].

اكر تقارن عضلات و موقعيت مفاصل نسبت به حالت اوليه

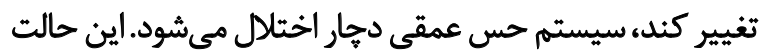

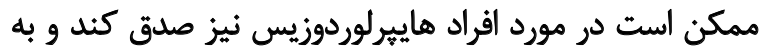

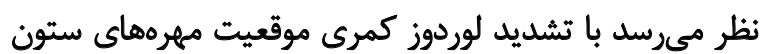

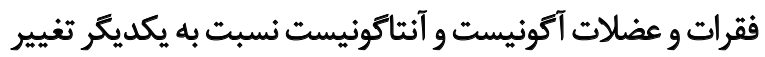

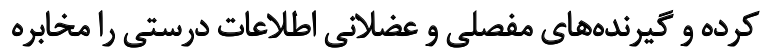

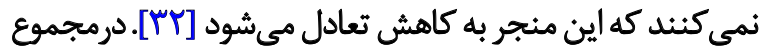

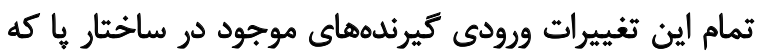

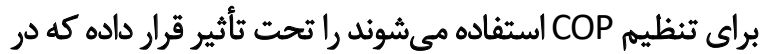

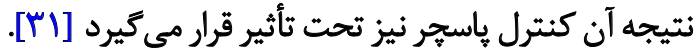
كاردوكى ^و همكاران به اين نتيجه رسيدند، در تعادل ايستا

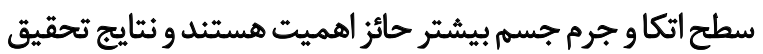

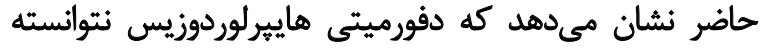

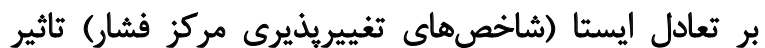

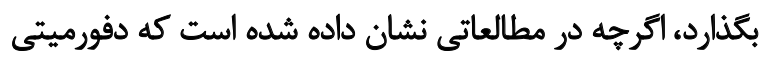

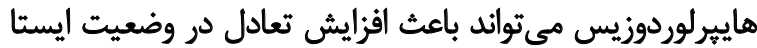

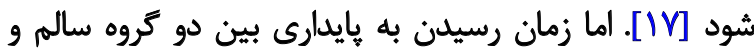

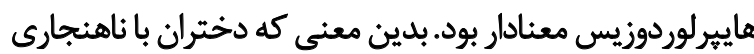

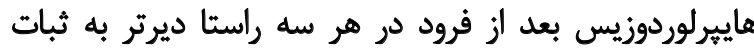

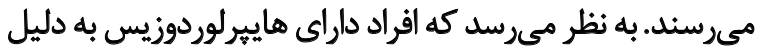

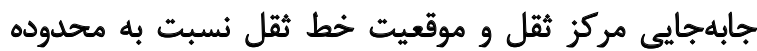

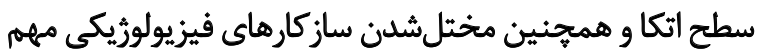

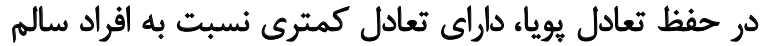

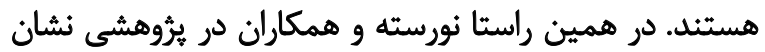

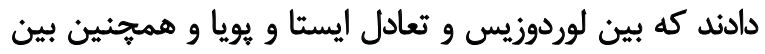

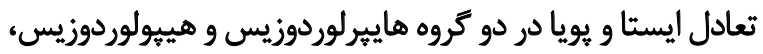
تفاوت معنادارى وجود ندارد [بr].

در تحقيقى ديكر محمدى و همكاران كزارش كردند كه اختلاف

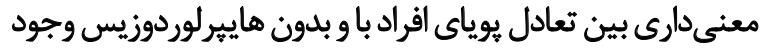

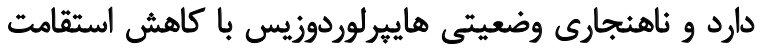

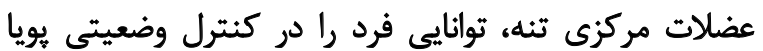

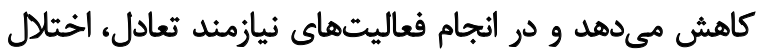

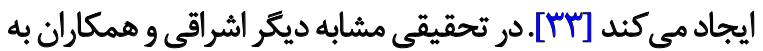




\section{References}

[1] Pavlovic A, Nichols DL, Sanborn CF, Dimarco NM. Relationship of thoracic kyphosis and lumbar lordosis to bone mineral density in women. Osteoporosis International. 2013; 24(8):2269-73. [DOI:10.1007/ s00198-013-2296-7] [PMID]

[2] Brianezi L, Cajazeiro DC, Maifrino LBM. Prevalence of postural deviations in school of education and professional practice of physical education. Journal of Morphological Sciences. 2011; 28(1):35-6.

[3] Bae TS, Mun M. Effect of lumbar lordotic angle on lumbosacral joint during isokinetic exercise: A simulation study. Clinical biomechanics (Bristol, Avon). 2010; 25(7):628-35. [DOI:10.1016/j.clinbiomech.2010.04.004] [PMID]

[4] LetafatKar A, Abdolvahabi Z. [General reform movement along with corrective exercises (Persian)]. Tehran: Avaye Zohur; 2011.

[5] Clark M, Lucett S. NASM essentials of corrective exercise training. Philadelphia: Lippincott Williams \& Wilkins; 2010.

[6] Magee DJ. Orthopedic physical assessment. $4^{\text {th }}$ Edition. Philadelphia, Pennsylvania, United States: Walter Burns Saunders; 2006.

[7] Murphy DF, Connolly DA, Beynnon BD. Risk factors for lower extremity injury: A review of the literature. British Journal of Sports Medicine. 2003; 37(1):13-29. [DOI:10.1136/bjsm.37.1.13] [PMID] [PMCID]

[8] Riemann BL, Lephart SM. The Sensorimotor System, Part II: The Role of Proprioception in Motor Control and Functional Joint Stability. Journal of Athletic Training. 2002; 37(1):80-4. [PMCID] [PMID]

[9] Nicolas V, Nicolas $P$, Jacqes V. Postural control during quit standing following cervical muscular - fatigue: Effect of change in sensory input. Neuroscience Letters. 2005; 378(3):135-9. [DOI:10.1016/j.neulet.2004.12.024] [PMID]

[10] Olmsted LC, Cracia CR, Hertel J, Shultz SJ. Efficacy of the stare excursion balance tests in detecting reach deficits in subject with chronic ankle instability. Journal of Athletic Training. 2002; 37(4):501-506. [PMCID] [PMID]

[11] Widhe T. Spine: Posture, mobility and pain: A longitudinal study from childhood to adolescence. European Spine Journal. 2001; 10(2):118-23. [DOI:10.1007/s005860000230] [PMID] [PMCID]

[12] Nazarian AB, Daneshjoo A, Ghorbani L, Ghaedi H. [The prevalence of lordotic and kyphotic deformities among different age groups (Persian)]. Journal of Research in Rehabilitation Sciences. 2009; 5(1):24-32

[13] Nault ML, Allard P, Hinse S, Blanc RL, Caron O, Labelle H. Relation between standing stability and body posture parameters in adolescent idiopathic scoliosis. Spine. 2002; 27(17):1911-7. [DOI:10.1097/00007632-200209010-00018] [PMID]

[14] Mackenzie WG. Sampath JS, Kruse R, Sheir-Neiss GJ. Backpacks in children. Clinical Orthopaedics and Related Research. 2003; 409:7884. [DOI:10.1097/01.blo.0000058884.03274.d9] [PMID]

[15] Murray HC, Elliott, C. Barton SE, Murray A. Do patients with ankylosing spondylitis have poorer balance than normal subjects. Rheumatology (Oxford). 2000; 39(5):497-500. [DOI:10.1093/rheumatology/39.5.497] [PMID]

[16] Aydog E, Depedibi R, Bal A, Eksioglu E, Unlü E, Cakci A. Dynamic postural balance in ankylosingspondylitis patients. Rheumatology (Oxford). 2006; 45(4):445-8. [DOI:10.1093/rheumatology/kei192] [PMID]
[17] Gardocki RJ, Watkins RG, Williams LA. Measurements of lumbopelvic lordosis using the pelvic radius technique as it correlates with sagittal spinal balance and sacral translation. The Spine Journal. 2002; 2(6):421-9. [DOI:10.1016/S1529-9430(02)00426-6]

[18] Gribble PA, Hertel J, Denegar CR, Buckley WE. The effect of fatigue and chronic ankle instability on dynamic postural control. Journal of Athletic Training. 2004; 39(4):321-9. [PMCID] [PMID]

[19] Shaw MY, Gribble PA, Frye JL. Ankle bracing, fatigue, and time to stabilization in collegiate volleyball athletes. Journal of Athletic Training. 2008; 43(2):164-71 [DOI:10.4085/1062-6050-43.2.164] [PMID] [PMCID]

[20] Wikstrom EA, Arrigenna MA, Tillman MD, Borsa PA. Dynamic pos tural stability in subjects with braced, functionally unstable ankle. Journal of Athletic Training. 2006; 41(3):245-50. [PMCID] [PMID]

[21] Gribble PA, Robinson RH. Alterations in knee kinematics and dy namic stability associated with chronic ankle instability. Journal of Athletic Training. 2009; 44(4):350-5. [DOI:10.4085/1062-605044.4.350] [PMID] [PMCID]

[22] Saidi F, Rajabi R, Ebrahimi TE, Mosavi SJ. [Reliability and validity of Iranian flexible ruler in lumbar spine curvature measurement (Persian)]. Journal of Sports Science and Medicine. 2009; 7(14):31-8.

[23] Hamill J, Knutzen KM. Biomechanical basis of human movement. $1^{\text {st }}$ ed. Philadelphia: Lippincott Williams \& Wikins; 1995.

[24] Gribble PA, Mitterholzer J, Myers AN. Normalizing considerations for time to stabilization assessment. Journal of Science and Medicine in Sport. 2012; 15(2):159-63. [DOI:10.1016/j.jsams.2011.07.012] [PMID]

[25] Fatahi F, Ghasemi Gh, Karimi MT. The effect of 8 weeks of core stability muscles training on kinetics of single-leg landing. Physical Treatments. 2016; 6(2):85-92. [DOI:10.18869/nrip.ptj.6.2.85]

[26] Bolgla LA, Uhl TL. Electromyographic analysis of hip rehabilitation exercises in a group of healthy participants. Journal of Orthopaedic \& Sports Physical Therapy. 2005; 35(8):487-94. [DOI:10.2519/ jospt.2005.35.8.487] [PMID]

[27] McBeth JM, Earl-Boehm JE, Cobb SC, Huddleston WE. Hip mus cle activity during 3 side-lying hip-strengthening exercises in distance runners. Journal of Athletic Training. 2012; 47(1):15-23. [DOI:10.4085/1062-6050-47.1.15] [PMID] [PMCID]

[28] Peterka RJ, Loughlin PJ. Dynamic regulation of sensorimotor integration in human postural control. Journal of Neurophysiology. 2004; 91(1):410-23. [DOI:10.1152/jn.00516.2003] [PMID]

[29] Regolin F, Carvalho GA. Relationship between thoracic kyphosis, bone mineral density, and postural control in elderly women. Brazilian Journal of Physiotherapy. 2010; 14(6):464-9. [DOI:10.1590/ S1413-35552010000600003] [PMID]

[30] Bruyneel AV, Chavet P, Bollini G, Allard P, Mesure S. The influence of adolescent idiopathic scoliosis on the dynamic adaptive behaviour. Neuroscience Letters. 2008; 447(2-3):158-63. [DOI:10.1016/j. neulet.2008.10.007] [PMID]

[31] Yalfani A, Anbarian M, Nikoo R, Anbarian M. [Relationship between postural control with sway- back malalignment in the non-athlete males (Persian)]. Scientific Journal of Ilam University of Medical Sciences. 2014; 22(5):189-201.

[32] Norasteh AA, Hosseini R, Daneshmandi H, Heidari SS. [Balance As sessment in Students with Hyperkyphosis and Hyperlordosis (Per sian)]. Sport Medicine. 2014; 6(1):57-71. 
[33] Mohammadi E, Balali Vashmesara J, Haddadnezhad M, Sanjari M. [Comparison of postural control and core endurance in young females with and without hyperlordosis (Persian)]. Journal of Sports Medicine and Physical Fitness. 2014; 1(2):109-28.

[34] Eshraghi A, Maroufi N, Sanjari MA, Keyhani MR, Saeedi H. [Static and dynamic balance of schoolgirls with hyperkyphosis (Persian)]. Scoliosis. 2009; 4(2): 05. [DOI:10.1186/1748-7161-4-S2-05] [PMCID] 